\title{
A lateral masking effect in tactile and blurred visual letter recognition
}

\author{
JACK M. LOOMIS \\ University of California, Santa Barbara, California 93106 \\ and \\ PATRICIA APKARIAN-STIELAU \\ Smith-Kettlewell Institute of Visual Sciences, Pacific Medical Center, San Francisco, California 94115
}

\begin{abstract}
In a letter recognition task in which the letters were presented temporally across two columns of a vibrotactile array, the masking effects of added columns of steady vibrotactile stimulation were investigated. The primary variable studied was the spatial separation between the masking columns and the letter-carrying columns. The results indicate a spatially dependent masking effect which is independent of the tactile masking associated with mask onset. A comparable masking effect was obtained with visual letter recognition using the same relative stimulus configurations viewed under conditions of optical blurring. An interpretation of the tactile masking is offered in terms of the limited spatial resolution of the cutaneous sense.
\end{abstract}

Although a good deal of research has been done on cutaneous sensitivity in the past, it was only recently that the advent of multiple stimulator systems made it possible to relate the facts of basic sensitivity to more complex perceptual functioning. In this connection, it is entirely reasonable that the phenomenon of cutaneous masking, evident when the detection threshold of one stimulator is raised in the presence of another stimulator, should manifest itself in tactile pattern recognition with multiple stimulator arrays. The present study is concerned with a form of spatial masking which has a demonstrable effect on letter recognition when the letters are presented temporally across a narrow display. Because this type of masking may be related to limited spatial resolution, we included for comparison corresponding visual conditions in which the letters and masks were optically blurred.

\section{METHOD}

\begin{abstract}
Approach
As in a previous study by the authors (Apkarian-Stielau \& Loomis, 1975), tactile and visual stimuli were presented using the $20 \times 20$ vibrotactile display and visual monitor of the Tactile Vision Substitution System (TVSS). Because only 16 or so of the columns of the tactile display make contact with the backs of most of the subjects, yet fairly large letters are needed to achieve a reasonable level of recognition accuracy, it was not feasible to
\end{abstract}

This work was supported by Department of Health, Education, and Welfare Grant 14-P-55282/9 from the Social and Rehabilitation Services, NIH Grant Research Support Grant 5 S01 RR05566, and the Smith-Kettlewell Eye Research Foundation. The authors are thankful to Irwin Pollack and to the reviewers for their helpful comments on the manuscript. These findings were reported in a paper presented at the 1975 meeting of the Psychonomic Society held in Denver, Colorado. activate columns of masking stimulation in addition to full size letters. We chose instead to present the letters temporally across two columns of the display and add additional columns of masking stimulation at varying separations from these video-controlled columns. This method of display (without the masking columns) is the stationary slit, moving-letter mode in the study by Loomis (1974), a mode of presentation which allows relatively high recognition accuracy for letters.

\section{Apparatus}

Tactile stimuli were presented to the backs of subjects using the dental chair version of the TVSS (Bach-y-Rita, 1972; Collins, 1970). Only the left half of the display was used, and subjects positioned themselves so that the right half of their backs made good contact with all 10 columns. When activated, all tactors, both masking and information-carrying ones, were run at the same nominal intensity, namely, $10 \mathrm{~dB}$ above threshold $(50 \mathrm{~mW})$. Vibration frequency, as always, was $60 \mathrm{~Hz}$. Vertical and horizontal spacing of the Teflon-tipped tactors is $12 \mathrm{~mm}$. In all conditions, only the two central columns of the 10-column submatrix were videc-controlled. Temporal presentation of a letter across these columns was accomplished by moving the letter horizontally in front of the stationary camera using a simple moving belt apparatus. Columns of masking stimulation were activated at the appropriate times in the masking conditions by throwing three-position toggle switches (off, on, and videocontrolled).

Blurred visual stimuli were produced in essentially the same fashion as before (Apkarian-Stielau \& Loomis, 1975). The light bulbs of the visual monitor are illuminated whenever the corresponding tactors are activated, either by video or manual control. These bulbs are enclosed in white plastic covers, $15 \mathrm{~mm}$ square, with the horizontal and vertical separation between covers (center to center) being $22 \mathrm{~mm}$. The visual display was covered by a sheet of white Plexiglas which was used to diffuse the image. The subject monocularly viewed this diffused image through a $3-\mathrm{mm}$ artificial pupil and a pair of positive spherical lenses $(12 \mathrm{D}+20 \mathrm{D})$ mounted in a pair of trial frames; the spacing between the cornea and the artificial pupil was about $2 \mathrm{~cm}$. The luminance specification of the stimulus on the diffusing screen is given in the previous paper. The present study differs a little from the previous one in that the letters were 18 points high rather than 9 points and, if displayed full field, 13 points wide rather than 7 ; 
thus, in this study, we presented both visual and tactile stimuli with the same zoom setting of the camera. These somewhat larger letters were viewed at a distance of $5.2 \mathrm{~m}$ rather than the distance of $3.0 \mathrm{~m}$ used previously. With these compensating changes, the specification of the visual stimulus for the observer is essentially the same as in the previous work. The subjective blur circle corresponding to a single bulb in the visual display was $6.7^{\circ}$ in diameter. Two columns of stimulation 18 rows high (corresponding to the vertical stroke of a letter like $F$ ) subtended $.44^{\circ} \times 4.35^{\circ}$ at the eye and because of the blurring devices gave rise to a blur pattern $6.9^{\circ} \times 11.1^{\circ}$.

In order to have some idea of the relative values of tactile and visual spatial resolution under these conditions, we presented stationary vertical and horizontal "square-wave gratings" of varying bar widths on the tactile and visual displays (e.g., two columns on, two columns off, etc). A very rough determination was made of the minimum spacing of bars (equal to the bar width) needed to distinguish vertical and horizontal gratings. These can only be rough measures, for edge information can be used by the subject as an alternative cue in the visual task and local differences of stimulation along the irregular surface of the back can be used as a subtle cue in the tactile task. In the tactile determination, a minimum bar spacing of one to two rows or columns was needed (one cycle per $2.4 \mathrm{~cm}$ ). In the visual determination, a minimum bar spacing of two rows or columns was needed for resolution, corresponding to a minimum separable angle of $28^{\prime}$ or a visual acuity of $20 / 560$.

\section{Stimuli}

Four conditions were tested under each modality. The first was the no-mask condition in which the letter sweeps temporally over the two central columns without any masking stimulation present. Figure 1 shows four discrete frames from the sequential presentation of the letter $K$. The blur pattern portrays very schematically the outlines of the optical blur pattern of the visual stimulus and of the hypothesized neural representation of the tactile stimulus after neural spreading in the afferent pathways. All 26 capital letters were used in the study; except for I, the presentation time in this slit scanning mode was $1.0 \mathrm{sec}$.

The other three conditions presented various configurations of masking stimulation. They are depicted in Figure 2, which, like Figure 1, portrays very schematically the optical and presumed neural blur patterns. In addition to the masking columns, the stimulation corresponding to the third frame in the sequential presentation of $\mathbf{K}$ (Figure 1) is shown. The overlap of the blur patterns in the masking conditions is evident, although the blur depicted in the figure is less severe than was actually the case; on the other hand, not depicted is the informative internal distribution of luminance of neural activity. The slit presentation of the letters was identical in all four conditions, the only change being the configuration of masking columns.

The Mask 1 condition presented a column of masking stimulation on each side of the video-controlled columns with an intervening space of three silent columns. The Mask 2 condition presented two columns of masking stimulation on each side with a spacing of two columns. Lastly, the Mask 3 condition also presented two columns of masking on each side but with no intervening space.

\section{Subjects}

Two males and two females, all in their late $20 \mathrm{~s}$, served in the experiment as paid volunteers. All of them had 20/20 uncorrected vision, as confirmed by a brief examination with a Snellen eye chart.

\section{Procedure}

Each subject was run a total of 102 -h sessions in which he/she received one complete alphabet for each of the eight conditions (four tactile and four visual). The various conditions were

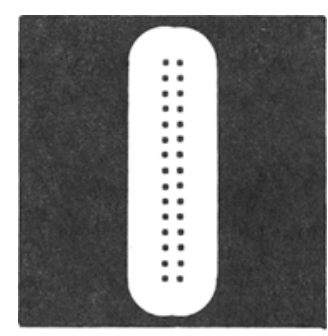

1

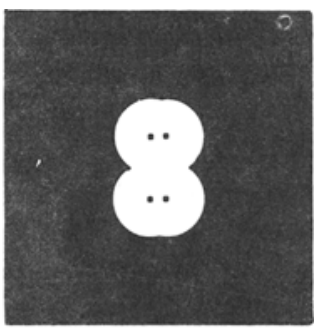

3

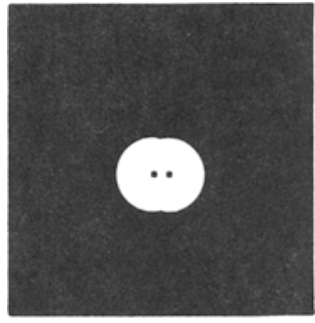

2

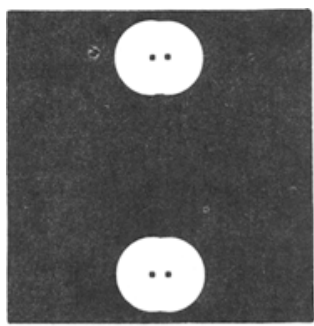

4
Figure 1. Four frames from the slit presentation of the letter $K$. The figure portrays very schematically the optical blur pattern or hypothesized pattern of cutaneous neural activity in each frame; it does not depict the internal distribution of luminance or of neural activity.

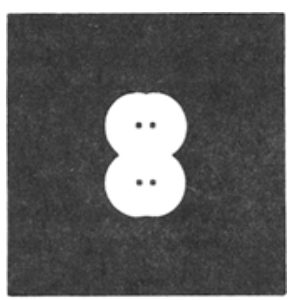

NO MASK

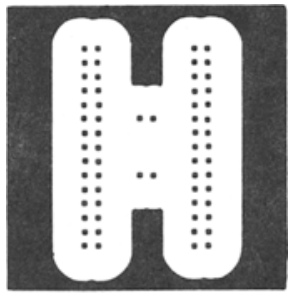

MASK 2

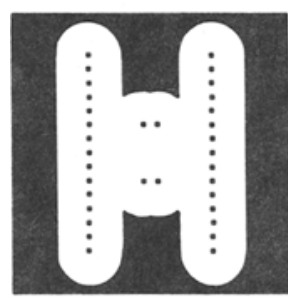

MASK 1
Figure 2. Schematic representation of the blur patterns corresponding to Frame 3 in the sequential presentation of the letter $K$ (see Figure 1) in each of the four conditions (refer to the text for description). The dots represent the light points or tactors activated. 
presented on a predetermined random schedule with a change of conditions every 13 letters.

For the visual masking conditions, the subject used the two symmetrically positioned masks to center his/her fixation before the letter presentation. For the visual no-mask condition, four fixation lights placed at the four corners of the 10-column submatrix were briefly illuminated just prior to the letter presentation and the subject used the four blur circles to center his/her gaze.

For the visual and tactile masking conditions, the masks were activated 4-5 sec before the letter presentation and remained on for several seconds after the presentation. We chose not to leave them on continuously between letters because of long-term tactile adaptation (Hahn, 1968; Jenkins \& Stone, 1941), but also wished to activate them sufficiently in advance to avoid the strong transient tactile (and visual) masking effects that occur with stimulus onset (Craig, 1974b; Gescheider, Herman, \& Phillips, 1970; Gilson, 1969a, b, 1974; Rollman, 1974; Schmid, 1961; Sherrick, 1964; Uttal, 1960).

The sequence of events during a recognition trial was as follows: a warning signal to the subject, activation of the masking columns (or brief illumination of the fixation lights in the visual no-mask condition), the letter presentation, cessation of the masking columns, the subject's response, and finally feedback to the subject on the correctness of his/her response.

\section{RESULTS}

The tactile and visual results are given in Figures 3 and 4 , respectively. Recognition accuracy, averaged over all four subjects, is presented for each of the 10 sessions as a function of masking condition. It is apparent that there is a consistent ordering of the four conditions throughout all 10 sessions for both tactile and visual recognition, with the no-mask condition being the easiest and the masking conditions increasing in difficulty from Mask 1 to Mask 3. The analyses to follow were based on the last five sessions, by which time performance had more or less stabilized after the initial learning period.

Recognition accuracy (percent correct), averaged over subjects for the last five sessions, is shown in Figure 5 as a function of masking condition and

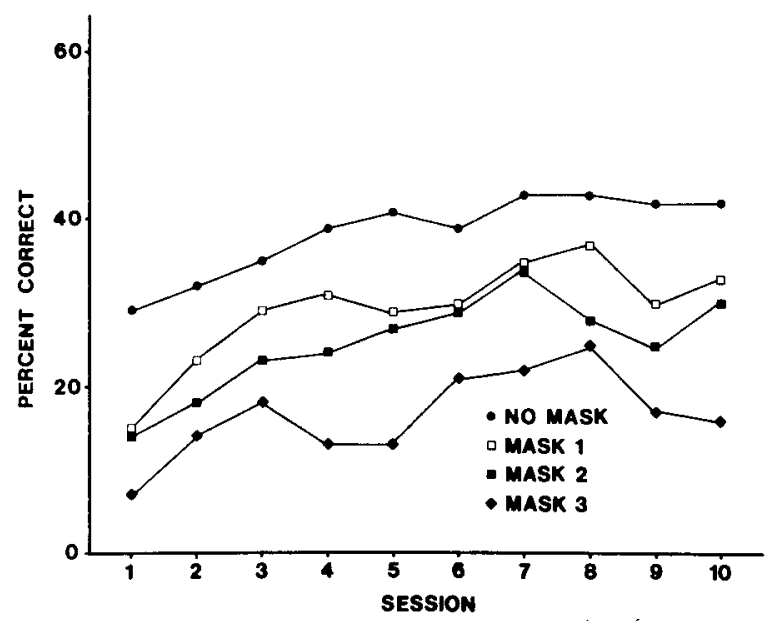

Figure 3. Tactile results. Recognition accuracy (percent correct) as a function of masking condition and session, averaged over subjects.

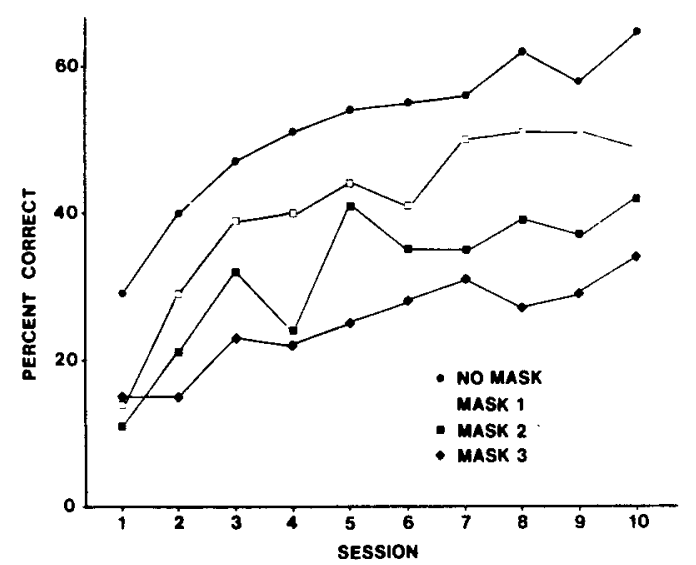

Figure 4. Visual results. Recognition accuracy (percent correct) as a function of masking condition and session, averaged over subjects.

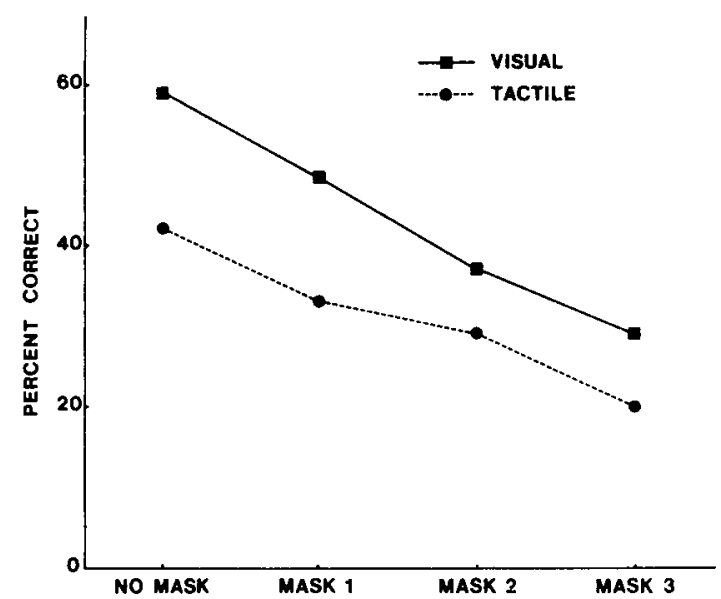

Figure 5. Average results for the last five sessions. Recognition accuracy (percent correct) as a function of masking condition and modality, averaged over subjects.

modality. A three-way analysis of variance (Guenther, 1964, pp. 132-133) showed the obvious downward trend as a function of masking condition to be significant at the .001 level $(F=33.9$, $\mathrm{df}=3 / 9)$. In addition, there was a significant Subjects effect $(\mathrm{F}=58.8, \mathrm{df}=3 / 128, \mathrm{p}<.001)$ and the following significant interactions: Modality by Condition $(\mathrm{F}=4.5, \mathrm{df}=3 / 9, \mathrm{p}<.05)$, Modality by Subject $(F=15.8, \mathrm{df}=3 / 128, \mathrm{p}<.001)$, and Condition by Subject $(F=2.7, \mathrm{df}=9 / 128, \mathrm{p}<.01)$.

In order to ascertain which differences between conditions were significant, we further analyzed the data using the Neuman-Keuls procedure (Winer, 1962 , p. 77). Because of the significant Condition by Modality interaction, we chose to use the error terms obtained from separate two-way analyses of variance based, respectively, on the tactile and visual data. For the tactile modality, the difference between Mask 1 and Mask 2 was nonsignificant; the difference between Mask 2 and Mask 3 was significant 
at the .05 level; and all other differences between pairs of conditions were significant at the .01 level. For the visual modality, the differences between no mask and Mask 1 and between Mask 2 and Mask 3 were significant at the .05 level; all other differences between pairs were significant at the .01 level.

The existence of a significant Modality by Condition interaction implies that the two masking functions are not identical for the two modalities. However, when compared in terms of ratios, the curves are considerably more alike, with performance ranging over a factor of two.

The fact that the two curves assume different absolute levels is merely a consequence of our failure to more evenly match the visual performance to the tactile performance for each subject. For two of our subjects, the match was quite good and for the other two, only fair.

As in previous work, we have also compiled the recognition accuracy for each letter of the alphabet as a function of modality and masking condition. Figure 6 gives the tabulations from this experiment (last five sessions) and from one condition of an earlier study. The row labeled "Tactile" (1974) is the array of diagonal cell values, converted to percent correct, from the confusion matrix of an identical slit-scanning condition in a study by Loomis (1974, Figure 7). These values are included to show the remarkable similarity of those data and of the present data from the tactile no-mask condition, which is a replication with different subjects. The productmoment correlation between these two sets of values is an impressive .90 , indicating that the variations in letter-specific recognition accuracy are reliable.

We also computed correlation coefficients between corresponding visual and tactile masking conditions. The correlations obtained were: no mask: $r=+.68$; Mask 1: $r=+.73$; Mask 2: $r=+.49$; and Mask 3: $r=+.53$, all of which are significant at the .01 level. The lower correlations for the two most difficult conditions may reflect the reduced variations in tactile recognition values, many of which were 0 or close to it. Although the positive correlations between the visual and tactile scores in corresponding conditions suggest a common factor that is determining performance, the fact that these correlations fall short of the .90 correlation between two independent replications of the tactile no-mask condition indicates that there must also be other factors not common to the two modalities.

\section{DISCUSSION}

The results clearly demonstrate the existence of a form of tactile lateral masking in a recognition task. Moreover, the ordering of the four tactile conditions in terms of recognition accuracy indicates that the masking effect is spatially dependent; for

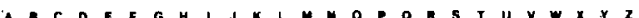

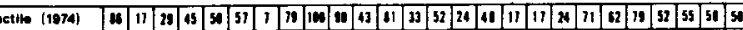

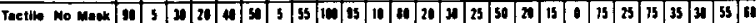
25 is 25 15 15 : $1: 50.45$

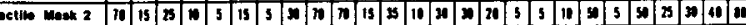

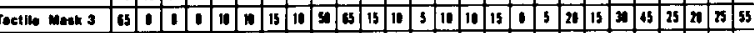

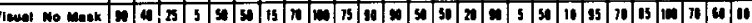

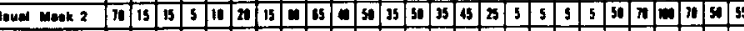

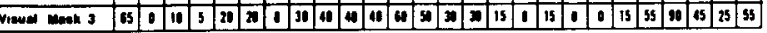

Figure 6. Recognition accuracy (percent correct) for each condition as a function of letter. Tactile (1974) refers to results from a previous experiment by Loomis (1974).

example, the double masks without any intervening space (Mask 3) gave rise to significantly lower recognition accuracy than did either of the configurations with an intervening space (Mask 1 and Mask 2). In addition to showing that this form of masking depends upon spatial proximity, this result rules out the possibility that the sole effect of the masks is one of diverting attention from the letter-carrying columns.

It is of critical importance that in the experimental procedure we activated the masking columns 4-5 sec prior to the letter presentation, for it means that the masking effect here is independent of the strong transient masking associated with onset of the masks, an effect well studied in the tactile masking literature.

With regard to psychophysical studies of masking with steady stimulation, the literature is very sparse; one such experiment was reported by Sherrick (1964), in which he investigated the threshold elevation of a vibratory test stimulus, $350 \mathrm{msec}$ in duration and positioned on the right index finger, produced by a vibratory masking stimulus, either steady or pulsed in simultaneity with the test stimulus and positioned either on the right little finger or left index finger. Although there was a degee of ipsilateral masking and a lesser degree of contralateral masking, neither effect was sizable in comparison with the masking associated with mask onset. However, it still may be the case that the effect Sherrick was investigating is not the essential cause for the decline in recognition accuracy in the present study. The interpretation we offer below considers the lateral masking here to be a consequence of the limited spatial resolution of the back. If this interpretation should be correct, then the masking effects Sherrick observed with the fingers would appear to be something quite different; relative to the respective measures of spatial resolution, Sherrick's stimulators on separate fingers were orders of magnitude more widely spaced than adjacent stimulators in our array placed against the back. If an experiment like the present one were to be done using the fingers, it would have to be done with a vibrotactile array, like that of the Optacon, having a stimulator spacing comparable to the spatial resolution of the finger.

The interpretation we offer for our results follows that given in our previous work (Apkarian-Stileau, 1975; Loomis, 1974). We suggest that the lateral 
masking effect observed here may in large part be a consequence of the poor spatial resolution of the back, which presumably reflects the neural organization of the cutaneous system and perhaps some mechanical wave spreading in the layers of the skin. In this view, this spatial masking is almost a trivial consequence, for to the extent that a pattern exceeds the spatial resolution of the sensory system, there is a lowering of the effective contrast of elements of the pattern. In the context of this experiment, the spreading stimulation of the masking columns, particularly in the close spacing condition (Mask 3), reduces the effective contrast of the letter-carrying columns, thus making perceptual registration of that information more difficult, with a consequent lowering of recognition accuracy.

To add force to this line of reasoning, we included the blurred visual conditions in the experiment. Simply by blurring the visual display, a demonstrable lateral masking effect was obtained ${ }^{1}$; in the visual case, it is quite apparent that the spreading light from the masking columns veils the letter-carrying columns, thus lowering the contrast of stimulation there. In principle, then, lateral masking should occur when spatial resolution of the sensory system is exceeded by the stimulus configuration. ${ }^{2}$

That this may, in fact, be the basis for the tactile results is suggested by the roughly comparable tactile and visual masking effects when the same stimulus configurations were presented to the two modalities under conditions of approximately the same spatial resolution (keeping in mind that the determinations of spatial resolution in the Method section should be treated only as approximate values). In addition, the positive correlations between the patterns of correct responses for corresponding tactile and visual conditions are further evidence that limited resolution is a common factor to both tasks. It should be said, however, that neither of the above points argues decisively for the limited resolution hypothesis; each point, like arguments presented in previous papers, is merely consonant with this interpretation. As a possible alternative explanation, one might argue that the tactile masking here reflects a suppression of the letter-carrying stimulation resulting from lateral inhibition by the surrounding masks. We have yet to devise an experimental test for deciding between these alternative interpretations.

Although our experimental procedure permitted us to study this lateral masking effect independently of the transient masking associated with mask onset as well as perhaps other spatiotemporal interactions, it at the same time limits the generality of our findings. In the normal usage of an optical-to-tactile conversion device, such as the Optacon or the TVSS, patterns usually move across the display in "TimesSquare" fashion. It is most probable that the spatial masking effect we have demonstrated contributes in part to the difficulty subjects have in recognizing patterns with this mode of presentation; for example, one of us found in an earlier study (Loomis, 1974) that moving a letter across just two columns of the tactile display led to superior recognition performance than when the full 20 columns were used. This result is quite amenable to interpretation in terms of lateral spreading of stimulation. On the other hand, it is just as probable that transient masking and other spatiotemporal interactions also contribute to the difficulty of tactile recognition when the entire display is modulated in time as well as in space; precisely this view has been taken by Craig in interpreting some recent masking effects he observed in letter recognition studies using the Optacon in the "Times-Square" mode (Craig, Note 1). Previously, too, the various forms of spatiotemporal interaction have certainly been of major concern to those concerned with the perception of patterns using multiple stimulator arrays (Bliss, Crane, Link, \& Townsend, 1966; Gilson, 1969b; Hill \& Bliss, 1968).

Realizing that these other masking effects may be present, we nevertheless think that our finding of a spatial masking effect and its interpretation are useful in understanding other tactile phenomena. One such phenomenon is the difficulty of perceiving "internal detail." In previous work with the TVSS (Scadden, 1971), it has been consistently observed that subjects have difficulty perceiving patterned stimulation or the absence of stimulation within a surrounding area of stimulation. This difficulty in perceiving internal detail is also commonly observed with the Optacon (Bliss, Note 2); it has also been reported by Craig (1974a, p. 82) in his work with a device very similar to the TVSS. In our own work, when the subject is given manual control of the camera, he/she will usually zoom in on the pattern so that only the internal detail or the outline of the area of no stimulation is present on the display. A related phenomenon occurs when the subject scans the external edges of a pattern; he/she will typically scan the outline of the pattern for distinguishing features, keeping the major part of the form off the display. Both strategies allow the subject to isolate the features of interest, thus preventing the other parts of the form from masking them.

\section{REFERENCE NOTES}

1. Craig, J. C. Vibrotactile letter recognition: The effects of a masking stimulus. In preparation.

2. James Bliss, personal communication.

\section{REFERENCES}

Apkarian-Stielau, P., \& Loomis, J. M. A comparison of tactile and blurred visual form perception. Perception \& Psychophysics. 1975, 18. 362-368. 
BACH-Y-RITA, P. Brain mechanisms in sensory substitution. New York: Academic Press, 1972.

Bliss, J. C., Crane, H. D., Lind, S. W., \& Townsend, J. T. Tactile perception of sequentially presented spatial patterns. Perception \& Psychophysics, 1966, 1, 125-130.

Collins, C. C. Tactile television-mechanical and electrical image projection. Institute of Electrical and Electronics Engineers Transactions on Man-Machine Systems, 1970, MMS-11, 65-71.

Crarg, J. C. Pictorial and abstract cutaneous displays. In F. A. Geldard (Ed.), Cutaneous communication systems and devices. Austin, Tex: Psychonomic Society, 1974. (a)

CraIG, J. C. Vibrotactile difference thresholds for intensity and the effect of a masking stimulus. Perception \& Psychophysics, 1974, 15, 123-127. (b)

Gescheider, G. A., Herman, D. D., \& Phillips, J. N. Criterion shifts in the measurement of tactile masking. Perception \& Psychophysics, 1970, 8, 433-436.

GilsoN, R. D. Vibrotactile masking: Some spatial and temporal aspects. Perception \& Psychophysics, 1969, 5, 176-180. (a)

GiLsON, R. D. Vibrotactile masking: Effects of multiple maskers. Perception \& Psychophysics, 1969, 5, 181-182. (b)

Gilson, R. D. Vibratory masking. In F. A. Geldard (Ed.), Cutaneous communication systems and devices. Austin, Tex: Psychonomic Society, 1974.

Guenther, W. C. Analysis of variance. Englewood Cliffs, N.J: Prentice-Hall, 1964.

HAHN. J. F. Tactile adaptation. In D. R. Kenshalo (Ed.), The skin senses. Springfield, Ill: Thomas, 1968.

Hill, J. W., \& BuIss, J. C. Modeling a tactile sensory register. Perception \& Psychophysics, 1968, 4, 91-101.

Jenkins, W. L., \& Stone L. J. Recent researchers in cutaneous sensitivity: II. Touch and the neural basis of the skin senses. Psychological Bulletin, 1941, 38, 69-91.

Loomis. J. M. Tactile letter recognition under different modes of stimulus presentation. Perception \& Psychophysics, 1974, 16, 401-408.
Rollman, G. B. Electrocutaneous stimulation. In F. A. Geldard (Ed.), Cutaneous communication systems and devices. Austin, Tex: Psychonomic Society, 1974.

SCADDEN. L. A. Perception of patterned vibratory stimulation: An evaluation of the Tactile Vision Substitution System. Doctoral thesis, University of the Pacific, 1971.

ScHMID, E. Temporal aspects of cutaneous interaction with twopoint electrical stimulation. Joumal of Experimental Psychology, $1961,61,400-409$.

SherRick, C. E. Effects of double simultaneous stimulation of the skin. American Journal of Psychology, 1964, 77, 42-53.

UTTAL. W. R. Inhibitory interaction of responses to electrical stimuli in the fingers. Joumal of Comparative and Physiological Psychology, 1960, 53, 47.51.

WINER, B. J. Statistical principles in experimental design. New York: McGraw-Hill, 192.

\section{NOTES}

1. The fact that the highest recognition accuracy in this experiment was only $59 \%$ is not a consequence of the cognitive demands of integrating over time with the slit mode of presentation. In an unpublished experimient where subjects foveally viewed the visual display in sharp focus, recognition was nearly perfect for all letters.

2. In a visual letter recognition study by the first author, now in preparation, a sizable lateral masking effect was obtained with sharp foveal viewing when the block capital letters, in isolation, approached the limit of foveal resolution. Adding various configurations of stimulation around the letters produced a substantial decline in recognition accuracy, even with moderate separation between letter and surround.

(Received for publication December 29, 1975; revision accepted August 5, 1976.) 